\title{
Intraoperative and Postoperative Complications after Arthroscopic Coracoclavicular Stabilization
}

\author{
Tae Kang Lim, MD, Whang Kyun Oh, MD \\ Department of Orthopaedic Surgery, Eulji Hospital, Eulji University School of Medicine, Seoul, Korea
}

Background: Arthroscopic stabilization of torn coracoclavicular (CC) ligaments gained popularity recently. However, loss of reduction after the operation and complications unique to this technique involving tunnel placement through the distal clavicle and coracoid process are concerns. The purpose of this study was to report intraoperative and early postoperative complications associated with this procedure.

Methods: This study retrospectively evaluated 18 consecutive patients who had undergone arthroscopic stabilization for torn CC ligaments between 2014 and 2015. The indications for surgery were acute or chronic acromioclavicular dislocation and acute fracture of the distal clavicle, associated with CC ligament disruption. Clinical outcomes were evaluated with the American Shoulder and Elbow Surgeons (ASES) and the University of California, Los Angeles (UCLA) scores. Intra- and postoperative complications and reoperations were investigated.

Results: There were six female and 12 male patients with a mean age of 47 years (range, 22 to 86 years). At a mean follow-up of $17 \pm 10$ months (range, 10 to 28 months), the mean ASES score was $88.8 \pm 19.9$ and the mean UCLA score was $30.9 \pm 5.2$. Intraoperatively, seven complications developed: breach of lateral cortex of the coracoid process in five patients, medial cortex of the coracoid process in one, and anterior cortex of the clavicle in one. Postoperative complications developed in eight patients: four ossifications of the CC interspace, four tunnel widening of the clavicle, one bony erosion on the clavicle, and one superficial infection. A loss of reduction was found in six patients. Reoperation was performed in three patients for loss of reduction in two and superficial infection in the other.

Conclusions: Arthroscopic CC stabilization resulted in high rates of intraoperative and early postoperative complications. Most of them were related to the surgical technique involving bone tunnel placement in the coracoid process and the clavicle.

Keywords: Coracoclavicular, Ligaments, Acromioclavicular, Dislocation, Arthroscopic

Since coracoclavicular (CC) ligaments have been known to play an important role in the acromioclavicular (AC) joint stability, procedures for fixation or reconstruction of the CC ligament have become popular surgical methods for treating AC joint dislocation. Beginning with the Bosworth screw fixation, ${ }^{1)}$ the Weaver-Dunn technique,

Received August 30, 2018; Accepted October 12, 2018

Correspondence to: Tae Kang Lim, MD

Department of Orthopaedic Surgery, Eulji Hospital, Eulji University School of Medicine, 68 Hangeulbiseong-ro, Nowon-gu, Seoul 01830, Korea

Tel: +82-2-970-8036, Fax: +82-2-973-3024

E-mail: 20140149@eulji.ac.kr and tendon graft for reconstruction of the CC ligament have been traditionally used, while there is a growing trend of using hook plates in recent times. Whichever method is used, the basic goal is to induce healing of the ruptured CC ligaments by reducing and maintaining the CC interspace. However, various complications associated with existing surgical methods have been reported: the Bosworth screw fixation is susceptible to screw breakage and requires screw removal; the Weaver-Dunn technique has a high recurrence rate due to failed fixation, and the hook plate is often associated with subacromial erosion or resorption and requires a secondary removal procedure. Recently, the arthroscopic technique for CC ligament 
reconstruction has become popular. Under arthroscopic guidance, a bone tunnel that passes through the clavicle and coracoid process is created, after which the suture or tendon graft is passed through the tunnel, and a metallic button (cortical button) is used for fixation of the supraclavicular area and the subcoracoid surface. This technique has the advantages of being relatively minimally invasive and providing effective fixation of the CC distance without requiring surgery to remove the internal fixture. Its biomechanical excellence has been proven by recent articles, and several actual clinical cases with favorable outcomes have also been reported. ${ }^{2-5)}$ Moreover, the indication for this technique is expanding because the technique can be used for not only the AC joint dislocation, but also acute distal clavicle fractures with CC ligament disruption. ${ }^{6}$ However, it is technically demanding, and the process of creating an anatomically accurate bone tunnel through the distal clavicle and small coracoid process can be difficult. Recently, several studies reported outcomes focusing on complications of this procedure, ${ }^{7,8)}$ whereas no related report has been published in Korea.

The purpose of the present study was to analyze the postoperative clinical and radiological outcomes of patients who underwent arthroscopic CC fixation to investigate intra- and postoperative complications associated with this procedure. We hypothesized that this procedure would show a high frequency of intra- and/or postoperative complications associated with the surgical technique.

\section{METHODS}

The present study retrospectively analyzed 18 patients who underwent arthroscopic CC ligament reconstruction between 2014 and 2015. The indications for this procedure were (1) acute or chronic Rockwood type 3 or 5 AC joint dislocation and (2) acute distal clavicle fracture (Neer type IIb) with CC ligament disruption. For type 3 AC joint dislocation, conservative therapy was primarily chosen, but among young and active patients, those who selected surgery after discussing the advantages and disadvantages of conservative therapy versus surgery underwent selective surgery. The exclusion criteria of the study were patients with prior history of shoulder surgery or infection and those with a follow-up period shorter than 6 months. In the present study, surgery was performed on 18 consecutive patients who satisfied the surgical indications during the study period, and all patients who underwent surgery were included in the analysis. No patients were excluded from this study. Each patient was given full oral and written information about the study, and written informed consent was obtained by the examining orthopedic surgeon. We conducted this study in compliance with the principles of the Declaration of Helsinki. The protocol of this study was reviewed and approved by Institutional Review Board Committee of Eulji Hospital (IRB No. 201707-003).

\section{Surgical Technique}

All surgeries were performed by the single surgeon (TKL). The patients underwent surgery under general anesthesia in a beach chair position. The standard portals for shoulder arthroscopy were used. The posterior portal was created medially at approximately $2 \mathrm{~cm}$ below the posterolateral corner of the acromion, and a $30^{\circ}$ arthroscope was inserted into the glenohumeral joint through the posterior portal, after which the anterior portal was created in the rotator interval and used as the working portal. First, a $30^{\circ}$ arthroscope was used to ensure that no other lesions are in the glenohumeral joint, after which the coracoid process was exposed as the rotator interval tissue was removed using a radiofrequency ablator. A $70^{\circ}$ arthroscope was used to secure a field of view of the base of the coracoid process, and the center of the coracoid process was identified after confirming the distal, basal, medial, and lateral margins of the coracoid process. Subsequently, a transverse incision approximately $2 \mathrm{~cm}$ in length was made above the distal clavicle, and the clavicle was exposed by thoroughly debriding the soft tissue in this area, followed by reduction of the AC joint while observing the C-arm images. With the patient's elbow being supported, the superiorly displaced clavicle was manually reduced by applying pressure in a downward direction, and if the state of reduction was determined to be good under C-arm observation, a K-wire $(1.6 \mathrm{~mm})$ was passed through from the acromion to the clavicle to temporarily hold the reduced state of the AC joint. Subsequently, the area of attachment between the clavicle trapezoid and conoid ligaments was confirmed on the $\mathrm{C}$-arm image, after which the point approximately 3.5 $\mathrm{cm}$ medial from the lateral tip of the distal clavicle, which is the point that passes through the center of the two ligaments, was marked above the clavicle to establish the point where the guide pin would be inserted. A C-shaped drill guide was positioned under arthroscopic visualization from the posterior viewing portal, where the proximal arm of the drill guide was placed on the clavicle, and the distal arm of the drill guide was adjusted to aim the base and center of the inferior cortex of the coracoid process. Subsequently, a 2.4-mm guide pin was inserted along the drill guide so that the guide pin would pass through four cortical bones going from the upper clavicle to the bottom sur- 
face of the coracoid process. If the guide pin successfully passed through the base and center of the coracoid process that had been targeted, the tunnel was reamed using a 4.0$\mathrm{mm}$ cannulated reamer (Fig. 1A and B). After removing only the guide pin, a PDS II (polydioxanone; Ethicon, Somerville, NJ, USA) suture was inserted through a cannulated reamer, and the suture exiting from the base of the coracoid process was pulled out from the joint through the anterior portal. A FiberTape (Arthrex, Naples, FL, USA) suture with a cortical button attached (Fig. 1C) was connected to this suture to form a loop, which was pulled up to the supraclavicular area by passing through the bone tunnel created by the coracoid process and the clavicle retrogradely by the shuttle-relay method. While maintaining tension in the supraclavicular area, the cortical button (coracoid side) was positioned firmly on the cortex of the inferior coracoid process. After connecting another cortical button (clavicle side) to the FiberTape suture pulled up to the supraclavicular area, both ends of the FiberTape suture were tied into a knot to ensure firm fixation while confirming the state of the AC joint reduction under Carm observation. The temporary K-wire used for fixation was removed, after which $\mathrm{C}$-arm images were used for final check of the state of the AC joint reduction as well as reconfirming that the two cortical buttons were positioned properly and especially the coracoid side cortical button was properly attached inferior to the coracoid process (Fig. 1D). Subsequently, the wound was closed to complete the surgery. For postoperative rehabilitation, the patients wore a shoulder brace for 4 weeks; subsequently, they were allowed to move the orthosis and begin shoulder exercise. The patients were prohibited from lifting heavy objects until 3 months postoperatively and were allowed to participate in sports activities 6 months postoperatively.

\section{Radiographic Evaluations}

Radiographic analysis was performed using plain radio- graphs. Plain radiography on both clavicles, for anteroposterior (AP) and axial images of the clavicle, was performed without weight-bearing in all patients preoperatively, immediately postoperatively, and during follow-up. The CC distance was measured in the AP images of both clavicles. In the picture archiving and communication system (PACS; General Electric, Chicago, IL, USA) images, a vertical line was drawn upward from the apex of the coracoid process and the closest distance to the cortical bone in the infraclavicular area was defined as the CC distance; the CC distance was measured simultaneously in both the affected and unaffected sides. All measurements were performed by an orthopedic surgeon who did not participate in the diagnosis or surgery (WKO). Measurement was performed twice for each patient, and the mean values were used for analysis. The CC percentage was calculated to determine the increase of the CC distance in the affected side relative to that in the unaffected side; a minus value indicated an overreduction. Based on this information, each AC joint dislocation was assessed according to the Rockwood classification. In the postoperative follow-up images, "reduction loss" was defined as an increase in the CC percentage of $\geq 25 \%$ compared with that measured immediately after surgery. ${ }^{9)}$

\section{Clinical Analysis}

For shoulder function assessment, the shoulder range of motion in all patients was measured using the American Shoulder and Elbow Surgeons (ASES) and University of California, Los Angeles (UCLA) scores. In addition, the medical records, operation records, operation videos, and plain radiographs obtained during follow-up of the patients were used to identify postoperative complications and reoperation. For intraoperative complications, malposition of the bone tunnel and bone tunnel damage or fracture during suture passage were investigated. For postoperative complications, clavicular or coracoid process
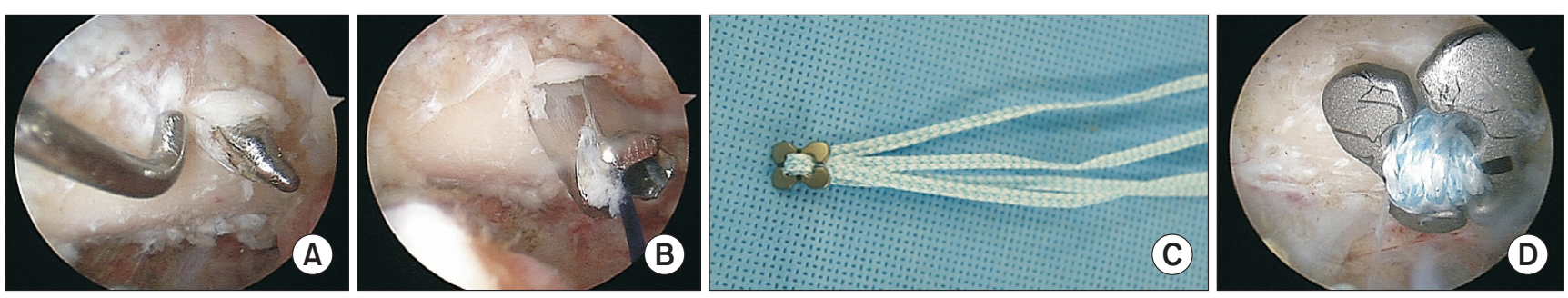

Fig. 1. (A) Arthroscopic coracoclavicular stabilization of the left shoulder in a 27-year-old male patient, viewing from posterior portal. A guide pin was drilled in the center and base of the inferior surface in the coracoid process. (B) The tunnel was made with a 4.0-mm cannulated reamer, and a suture was passed for shuttle relay though the reamer. The cortical button and FiberTape sutures were prepared before fixation (C) and properly placed on the subcoracoidal location (D). 
fracture through the tunnel, displacement of the cortical button and consequent reduction failure, tunnel widening, bone erosion due to cortical button, distal clavicle osteolysis or osteoarthritis, heterotopic ossification, nerve damage, and infection were investigated.

\section{Statistical Analysis}

CC distances measured preoperatively, immediately postoperatively, and during follow-up were compared using paired $t$-tests. Independent $t$-tests were performed to statistically evaluate the differences in the measured values between the groups with and without reduction loss and groups with and without complications other than reduction loss. The $p$-value of 0.05 was set as the level of statistical significance.

\section{RESULTS}

\section{Clinical and Radiographic Outcomes}

The patient population comprised six females and 12 males with a mean age of 47 years (range, 22 to 86 years). Eight patients sustained injury in their dominant hand side. The causes of injury included pedestrian traffic accidents $(n=3)$, motorcycle accidents $(n=4)$, bicycle accidents $(n=3)$, misstep accidents $(n=6)$, and sports-related injuries $(\mathrm{n}=2)$. With respect to the type of injury, 13 patients had an AC joint dislocation and five had a Neer type IIB distal clavicle fracture (Fig. 2); according to the Rockwood classification, there were 10 type 5 and 3 type 3 , respectively. The patient population included one patient with a chronic AC joint dislocation, whereas the rest had an acute injury. The patient with chronic injury was treated with autogenous palmaris longus tendon grafting in conjunction with cortical button and suture fixation. The mean follow-up period was $17 \pm 10$ months (range, 10 to 28 months).

Radiographic outcomes showed a decrease in the mean CC distance from a preoperative value of $17.1 \pm 3.4$ $\mathrm{mm}$ to a postoperative value of $9.8 \pm 4.0 \mathrm{~mm}(p<0.001)$, whereas the value at the final follow-up was $11.53 \pm 4.1$ $\mathrm{mm}$, which did not show a statistically significant difference compared with the immediate postoperative value $(p=0.103)$. The mean CC percentage at the preoperative, immediate postoperative, and final follow-up was $130 \% \pm$ $45 \%, 30 \% \pm 61 \%$, and $47 \% \pm 45 \%$, respectively. During the follow-up period, six patients (33\%) had reduction loss. The mean CC percentage in the group with reduction loss was larger than the group without reduction loss $(75 \% \pm$ $50 \%$ and $37 \% \pm 38 \%$, respectively) although this difference was not statistically significant $(p=0.095)$. In addition, bone union was achieved in all cases where surgery was performed on the fracture. With respect to clinical outcomes, the mean ASES and UCLA scores at the final follow-up was $88.8 \pm 19.9$ points and $30.9 \pm 5.2$ points, respectively. Based on UCLA scores, there were 15 excellent, one good, and two fair outcomes. On the comparison between the groups with and without reduction loss, no statistically significant differences were found in the mean ASES score $(92.0 \pm 4.6$ and $87.5 \pm 23.6$, respectively; $p=$ $0.222)$ and UCLA score ( $31.5 \pm 2.8$ and $30.8 \pm 6.0$, respectively; $p=0.256$ ).

\section{Complications}

Intraoperative complications occurred in seven patients
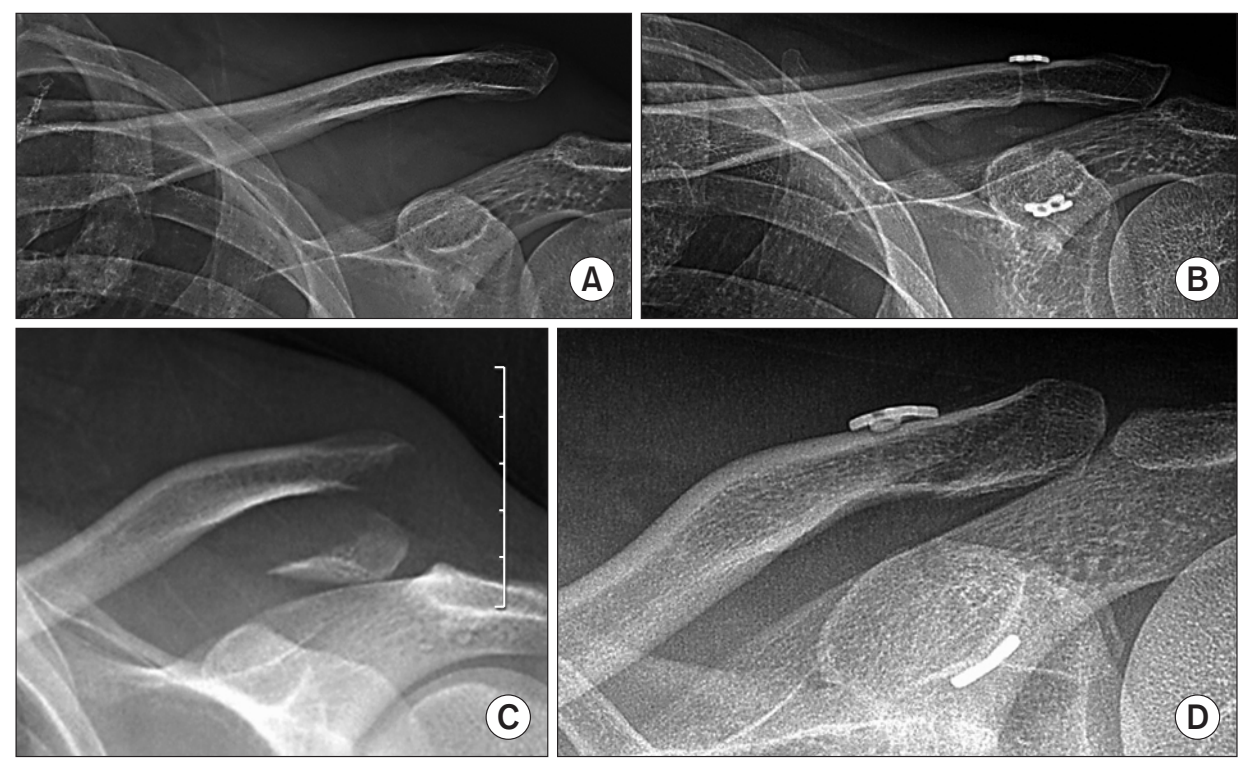

Fig. 2. Preoperative (A) and 24-month postoperative (B) radiographs of a 27-year-old patient with acromioclavicular dislocation of the left shoulder (Rockwood classification, type 5). Preoperative (C) and 6-month postoperative (D) radiographs of a 45-year-old male patient with a distal clavicle fracture of the left shoulder (Neer classification, type IIB). 
Lim and Oh. Complications after Arthroscopic Coracoclavicular Stabilization

Clinics in Orthopedic Surgery • Vol. 11, No. 1, 2019•www.ecios.org

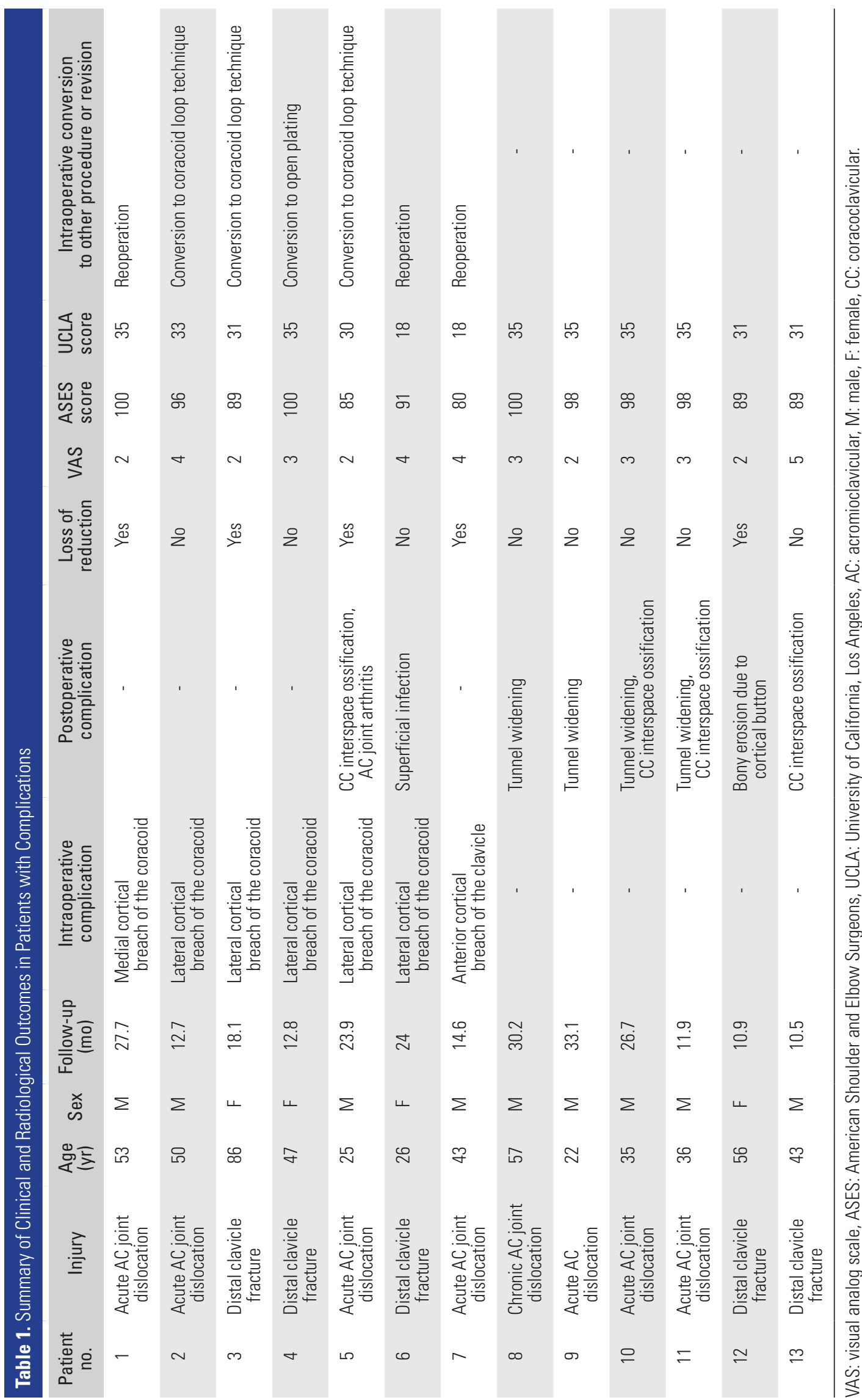


(39\%) (Table 1), which were all associated with the bone tunnel, including five patients with lateral cortex breach in the coracoid process during drilling or reaming and one patient with medial cortex breach (Fig. 3). In the remaining one patient, bone tunnel on the coracoid process side was formed properly, but the anterior cortex of the clavicle was damaged during the passage of FiberTape. Among the seven patients with bone tunnel damage, surgery was converted to fixation using the coracoid loop technique in four patients, in which fixation was performed by looping the FiberTape below the coracoid process instead of the coracoid bone tunnel (Fig. 4), whereas in one case of distal clavicle fracture, the method was changed to an open surgical procedure with plate fixation. On the comparison between the groups with $(n=7)$ and without intraoperative complications $(n=11)$, no statistically significant differences were found in the mean CC percentage at the final follow-up $(40.7 \% \pm 50.5 \%$ vs. $55.7 \% \pm 42.7 \%$, respectively; $p=0.443$ ). Meanwhile, comparisons of clinical scores between the groups with and without intraoperative complications showed a significant difference in the mean ASES score $(82.8 \pm 30.2$ and $92.9 \pm 6.2$, respectively; $p=$
0.047), while no significant difference was noted in the mean UCLA score (30.4 \pm 5.8 and $31.4 \pm 4.8$, respectively; $p=0.848)$.

With respect to postoperative complications, other than reduction loss, there were four cases of heterotopic ossification of the CC interspace, four cases of tunnel widening, one case of cortical bone erosion in the clavicle caused by the cortical button, one case of arthritis of the AC joint, and one case of superficial infection. Reoperation was performed in three patients, including two patients who underwent refixation with open surgery due to reduction loss and one patient with superficial infection involving skin irritation caused by the suture, for which infected tissue curettage and suture removal were performed. On the comparison between the groups with ( $\mathrm{n}$ $=9$ ) and without postoperative complications or reoperation $(\mathrm{n}=9)$, no statistically significant differences were found in the mean CC distance (10.5 \pm 3.4 and $12.4 \pm 4.6$ $\mathrm{mm}$, respectively; $p=0.489)$ and CC percentage $(45.8 \% \pm$ $45.3 \%$ and $53.0 \% \pm 47.1 \%$, respectively; $p=0.662$ ) at the final follow-up. The groups with and without postoperative complications or reoperation also showed no statisti-
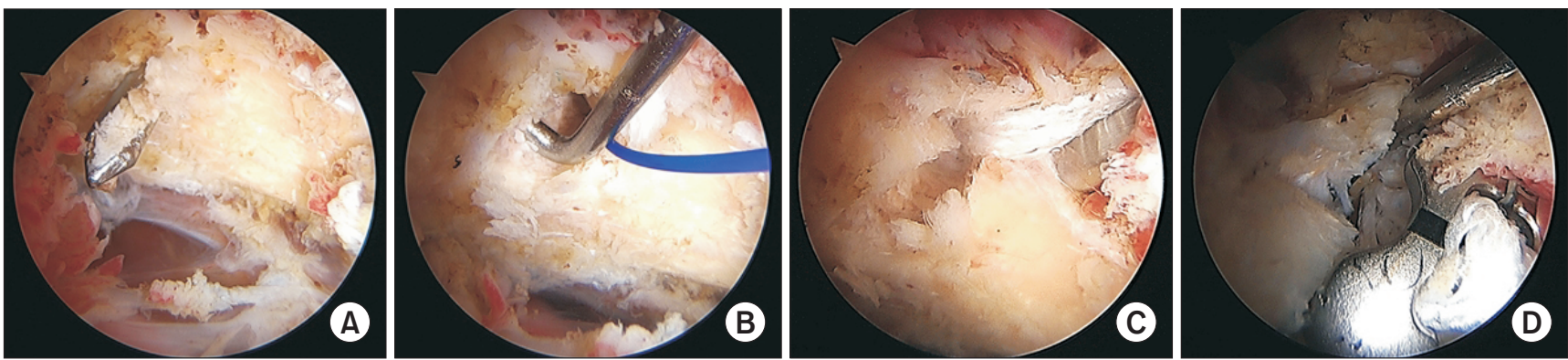

Fig. 3. (A) Arthroscopic image of the right shoulder in a 24-year-old female patient, viewing from posterior portal. A drill guide seemed to be placed properly into the base and center of the inferior surface in the coracoid process; however, lateral cortex breach of the coracoid process occurred after reaming (B) and the bone tunnel was further damaged during pull-out of the FiberTape sutures (C). (D) Arthroscopic image of the right shoulder of a 57-year-old female patient showing lateral cortex breach after placement of the cortical button and sutures.
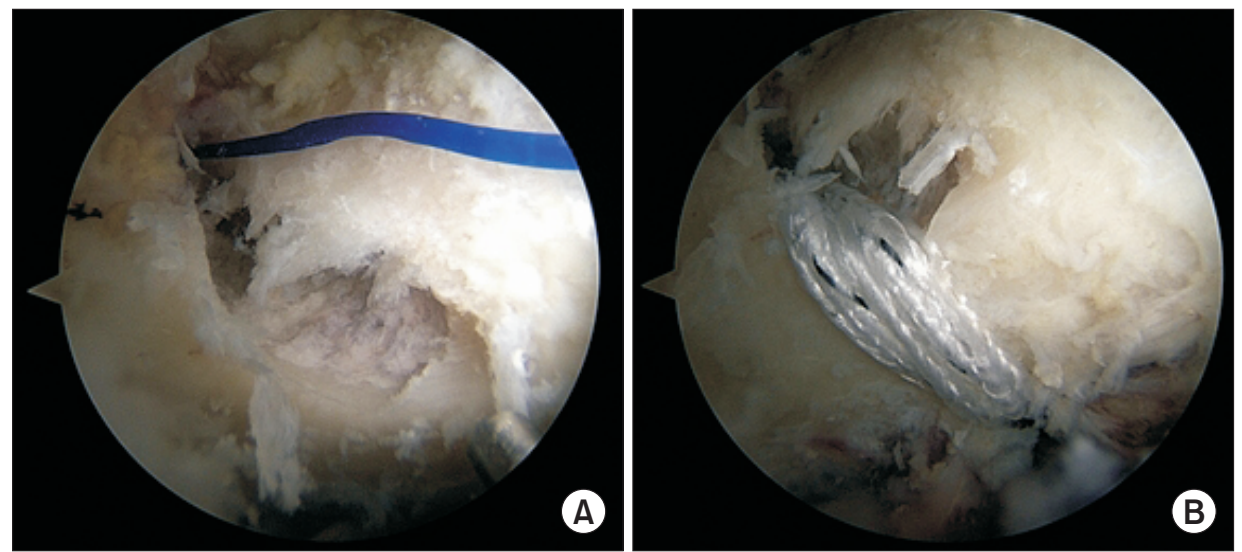

Fig. 4. Arthroscopic images of the right shoulder of a 28-year-old male patient, viewing from the posterior portal. (A) A lateral cortex breach occurred during reaming. (B) The fixation method was converted to the coracoid loop technique where the FiberTape was looped below the coracoid process instead of being passed through the coracoid bone tunnel. 
Lim and Oh. Complications after Arthroscopic Coracoclavicular Stabilization

Clinics in Orthopedic Surgery • Vol. 11, No. 1, 2019•www.ecios.org

cally significant differences in the ASES score $(84.7 \pm 28.5$ and $92.4 \pm 6.4$, respectively; $p=0.094$ ) and UCLA score (31.1 \pm 5.7 and $31.0 \pm 4.8$, respectively; $p=0.818$ ).

\section{DISCUSSION}

The key finding in the present study was that the frequency of perioperative complications was very high following arthroscopic CC ligament reconstruction. Among 18 patients, intraoperative and early postoperative complications occurred in seven and nine patients, respectively. These complications were unique because they were mostly associated with the process of creating a bone tunnel in the coracoid process. The surgical technique requires four cortical bones to be drilled all at once by starting from the supraclavicular area and accurately penetrating the base and mediolateral center of the inferior surface in the coracoid process while arthroscopically observing only the inferior surface of the coracoid process. We would like to emphasize that surgeons who plan to use this procedure should be aware of such limitations associated with the surgical technique.

Existing articles have reported on the biomechanical excellence and good clinical outcomes of this procedure, but several recent studies focused on the limitations and complications associated with this procedure. Shin and $\mathrm{Kim}^{8)}$ reported that complications occurred in eight of 18 cases (44\%) in CC reconstruction by arthroscopic TightRope, where most of the complications were associated with the surgical instruments and technique, including two cases of fixation failure on the coracoid process side and one case of distal clavicle fracture that passed through the clavicular tunnel area. Martetschlager et al. ${ }^{10)}$ also reported that the frequency of complications among 59 cases of CC ligament reconstruction was $27 \%$ (16/59), including one case of coracoid process fracture and two cases of clavicle fracture. In a study by Milewski et al., ${ }^{11)}$ complication was found in eight of 17 cases in which fixation was performed through the coracoid process tunnel, including two cases of coracoid process fracture and one case of failed cortical button fixation on the coracoid process side during surgery. In the present study, seven cases of intraoperative complications associated with bone tunnel were found. The most stable location of the bone tunnel is known to be the base and center of the inferior coracoid process, and even when a guide is used to set the exact target point, successfully drilling this area may be difficult at times. Moreover, even if the tunnel is created properly, the bone tunnel may be damaged during reaming or when a thick suture is passed through the tunnel. The risk of such an intraoperative complication may be even higher particularly in elderly patients or patients with poor bone quality. If such bone tunnel damage occurs during surgery, the surgical procedure that requires transcoracoid fixation must be given up. Instead, the surgery can be completed with a secondary procedure where the suture is looped inferiorly to the coracoid process and passed above the clavicle or the procedure can be changed to an open surgery in completing the surgery by fixation. In doing so, the operating time becomes longer, and the surgeon becomes more fatigued, and thus, such intraoperative complications associated with bone tunnel have some clinical significance.

The fact that postoperative reduction loss was observed in six of 18 cases in the present study should also be kept in mind. The reduction and maintenance of the $\mathrm{AC}$ joint dislocation by this procedure may have been inadequate. Among studies that used a similar arthroscopic procedure, Shin and $\mathrm{Kim}^{8)}$ reported that fixation failure with $\geq 50 \%$ increase in the CC distance during postoperative follow-up, compared with the initial fixed state, was found in six of 18 patients (33\%). Cook et al. ${ }^{7)}$ reported that among ten patients who underwent surgery, complete redislocation and subluxation occurred in five and three patients, respectively, mentioning that they are no longer using this procedure. As an open surgical technique, Choi et al. ${ }^{9)}$ used an autograft tendon instead of sutures for CC ligament reconstruction and found that reduction loss occurred in $47 \%$ of the cases, which indicated that frequency of reduction loss was high regardless of the material used in reconstruction. However, a recent study by Shin et al. ${ }^{5)}$ that used the same material as the present study reported much better outcomes with reduction loss occurring in only one of 21 patients. Such results may be attributed to creating two tunnels rather than just one in the clavicle, while allowing fixation with reconstruction of both the conoid and trapezoid ligaments that make up the CC ligament. Moreover, the repair or reconstruction of the AC ligament, not just the CC ligaments, should always be considered for stronger fixation.

The findings in the present study demonstrated that arthroscopic reconstruction of the CC ligament, which has become popular in recent times, has significant limitations. Alternatives for overcoming such limitations need to be developed, for which, a few modified versions of the current technique can be considered. The first method is suture and fixation without creating the coracoid process tunnel to prevent complications associated with the bone tunnel. Instead of drilling a bone tunnel in the coracoid process that is small in size, the method loops the suture 
around the inferior coracoid process and fixes it on the clavicle. Such method has been suggested, ${ }^{12)}$ but, there are concerns about a decrease in fixation force of the suture when movement of the suture in the subcoracoid occurs. The method of fixation with insertion of a suture anchor on the coracoid process for fixation on the coracoid process side can also be an alternative. However, this method also has the disadvantage of weak pull-out strength of the suture anchor, compared with the method of transcoracoid fixation. In addition, creating the bone tunnel is expected to be relatively easier with an open surgical technique than with the arthroscopic technique, and thus this can be considered as one of the clinical alternatives. Finally, the discussion on whether the technique used in the present study is the most anatomically ideal method is necessary. In a recent cadaveric study, Koh et al. ${ }^{13)}$ reported that when the bone tunnel was anatomically positioned along the direction of the conoid and trapezoid ligaments that make up the CC ligament, the actual bone tunnel was not positioned in the base and center of the coracoid process, as targeted by the current technique, but rather, there was medial cortical breach at the coracoid process. This raises concerns that the bone tunnel method currently used does not suit the purpose of the present study, which is to reconstruct the ligament in the most anatomically ideal location. Arthroscopic procedures still have many limitations, and thus continued research is needed to develop a technique that can reduce the frequency of reduction loss, have fewer complications, and ultimately enable reconstruction of the ligament with proper anatomical position.
There are some limitations of the present study. First, it was a retrospective study with a small sample size. Second, the study did not have a control group; hence, comparison could not be made against nonsurgical methods or other surgical methods. Finally, the high frequency of intraoperative complications, including the bone tunnel not being created properly, may be associated with poor surgical technique by the surgeon. Because it is an obvious fact that more experienced surgeons would produce different outcomes, the present study results should be cautiously interpreted. Despite this, the present study has clinical significance in that the findings may be used as reference data by novice surgeons who are attempting this technique. Finally, this study included five patients with distal clavicle fractures. The surgical techniques stabilizing the CC interspace were the same in both the AC joint dislocation and the fracture of distal clavicle (Neer type IIB). Therefore, the outcomes in those patients were also believed to be relevant to the purpose of the study focusing on intra- and postoperative complications related to the surgical technique.

Arthroscopic CC stabilization resulted in high rates of intraoperative and early postoperative complications. Most of them were related to the surgical technique involving bone tunnel placement in the coracoid process.

\section{CONFLICT OF INTEREST}

No potential conflict of interest relevant to this article was reported.

\section{REFERENCES}

1. Bosworth BM. Complete acromioclavicular dislocation. $\mathrm{N}$ Engl J Med. 1949;241(6):221-5.

2. Balog TP, Min KS, Rumley JC, Wilson DJ, Arrington ED. Arthroscopic anatomic coracoclavicular ligament repair using a 6-strand polyester suture tape and cortical button construct. Arthrosc Tech. 2015;4(6):e757-61.

3. Pennington WT, Hergan DJ, Bartz BA. Arthroscopic coracoclavicular ligament reconstruction using biologic and suture fixation. Arthroscopy. 2007;23(7):785.

4. Scheibel M, Ifesanya A, Pauly S, Haas NP. Arthroscopically assisted coracoclavicular ligament reconstruction for chronic acromioclavicular joint instability. Arch Orthop Trauma Surg. 2008;128(11):1327-33.

5. Shin SJ, Jeon YS, Kim RG. Arthroscopic-assisted coracoclavicular ligament reconstruction for acute acromioclavicular dislocation using 2 clavicular and 1 coracoid cortical fixation buttons with suture tapes. Arthroscopy. 2017;33(8):1458-66.

6. Blake MH, Lu MT, Shulman BS, Glaser DL, Huffman GR. Arthroscopic cortical button stabilization of isolated acute Neer type II fractures of the distal clavicle. Orthopedics. 2017;40(6):e1050-4.

7. Cook JB, Shaha JS, Rowles DJ, Bottoni CR, Shaha SH, Tokish JM. Early failures with single clavicular transosseous coracoclavicular ligament reconstruction. J Shoulder Elbow Surg. 2012;21(12):1746-52.

8. Shin SJ, Kim NK. Complications after arthroscopic coracoclavicular reconstruction using a single adjustable-looplength suspensory fixation device in acute acromioclavicular joint dislocation. Arthroscopy. 2015;31(5):816-24.

9. Choi NH, Lim SM, Lee SY, Lim TK. Loss of reduction and complications of coracoclavicular ligament reconstruction with autogenous tendon graft in acute acromioclavicular 
Lim and Oh. Complications after Arthroscopic Coracoclavicular Stabilization

Clinics in Orthopedic Surgery • Vol. 11, No. 1, $2019 \bullet$ www.ecios.org

dislocations. J Shoulder Elbow Surg. 2017;26(4):692-8.

10. Martetschlager F, Horan MP, Warth RJ, Millett PJ. Complications after anatomic fixation and reconstruction of the coracoclavicular ligaments. Am J Sports Med. 2013;41(12):2896-903.

11. Milewski MD, Tompkins M, Giugale JM, Carson EW, Miller MD, Diduch DR. Complications related to anatomic reconstruction of the coracoclavicular ligaments. Am J Sports
Med. 2012;40(7):1628-34.

12. Parnes N, Blevins M, Carey P, Carr B. Arthroscopic reconstruction of the coracoclavicular ligaments using a coracoid cerclage technique. Arthrosc Tech. 2016;5(2):e241-6.

13. Koh KH, Shon MS, Choi NH, Lim TK. Anatomic tunnel placement is not feasible by transclavicular-transcoracoid drilling technique for coracoclavicular reconstruction: a cadaveric study. Arthroscopy. 2018;34(7):2012-7. 